\title{
Working with natural processes to achieve sustainable habitat creation - Good Practice
}

\author{
Natalie Frost, ABP Marine Environmental Research Ltd., nfrost@abpmer.co.uk
}

\begin{abstract}
The number of managed realignment schemes being planned and implemented has increased markedly over the last decade and this growth can be expected to continue with the need for sustainable flood protection and habitat creation. The design of such schemes not only plays an important part in determining the potential impacts on the existing environment but can also be used to deliver maximum ecological and flood risk benefits within the newly created site. Where sites are developed to achieve maximum benefit from existing morphological, hydrodynamic and ecological features this may, for example, reduce costs in terms of flood defence through reduced requirements for hard engineering and ongoing maintenance. In addition an understanding of natural processes can help to deliver maximum ecological functioning and in turn delivery against biodiversity targets. Predicting the future habitat composition and distribution of schemes is also vital in understanding the sustainability of a site in response to future climate change projections. In this respect full consideration of natural processes can contribute significantly to the overall success of a scheme.
\end{abstract}

Habitat creation schemes are undertaken with a range of different objectives, which in turn forms an important consideration in the design phase of a project. Those schemes being undertaken with specific compensation objectives often require a degree of site manipulation and engineering to create specific habitats whilst still seeking to maintain sustainability. In contrast other schemes, have a greater emphasis on non-intervention, allowing natural processes to dominate site development. This is arguably a more sustainable approach to site development resulting in minimal intervention and correspondingly disturbance. This presentation will review the various stages of project design, focusing on habitat prediction methodologies, that can be applied to achieve best practice within managed realignment schemes. A series of case studies will be incorporated in order to provide lessons learnt from those schemes that have already been implemented. Each of these has been undertaken for different reasons and is accompanied by distinct site-specific issues which are associated with their objectives, location and their scale. This information will be drawn together along with additional material derived from the Online Managed Realignment Guide (OMReG) database to provide an overview of key lessons learned to date. OMReG serves as a 'collecting point' for project-specific information about coastal Managed Realignment and Regulated Tidal Exchange projects in the UK and Northern Europe. 